\title{
Frozen Section as a Guide in Intraoperative Decision-making in the Management of Adnexal Mass Lesions
}

\author{
Subbiah Shanmugam ${ }^{1}$, Gopu Govindasamy ${ }^{2}$, Sujay Susikar ${ }^{3}$, Prinith S Fells ${ }^{4}$
}

\begin{abstract}
Ovarian tumors present with vague pelvic, abdominal, and menstrual symptoms. The lack of histological diagnosis beforehand with no definite evidence of malignancy intraoperatively may put the surgeon at the crossroads, whether to proceed with a complete surgical staging or conservative approaches, such as a fertility sparing procedure or a simple ovariectomy. Frozen section done intraoperatively in the aforementioned setting may help in differentiating benign and invasive ovarian tumors and help in proceeding with an optimal surgical procedure. But the reliability of frozen is limited in borderline and mucinous ovarian tumors.

Keywords: Accuracy, Borderline tumors of ovary, Frozen section.

Journal of South Asian Federation of Menopause Societies (2019): 10.5005/jp-journals-10032-1181
\end{abstract}

\section{INTRODUCTION}

Ovarian tumors may present with vague pelvic, abdominal, and menstrual symptoms. The lack of histological diagnosis beforehand and no definite evidence of malignancy intraoperatively may put the surgeon at crossroads, on whether to proceed with a complete surgical staging or conservative approaches, such as a fertility sparing procedure by simple ovariectomy. Frozen section done intraoperatively may help in classifying tumors into benign, borderline, and invasive ovarian tumors and help in deciding with an optimal surgical procedure.

\section{Materials and Methods}

Patients diagnosed with ovarian tumors clinically and by imaging, with equivocal CA-125, without a preoperative histopathological diagnosis of malignancy were included in the study. These patients underwent excision of adnexal lesions which were then subjected for frozen examination. The objective of this study is to assess the accuracy, reliability, and limitations of the frozen section in the diagnosis of adnexal mass lesions by comparing it with final histopathological diagnosis. The concordance and discordance of the frozen section with the final histopathological diagnosis of 46 adnexal mass lesions were studied. Sensitivity, specificity, and accuracy rates were determined and results analyzed.

\section{Results}

Totally, 46 patients were included in the study, of which 17 patients were benign, 7 patients were borderline, and 22 patients were malignant as per final histopathological diagnosis (Table 1).

Among the benign tumors, 16 patients were diagnosed as benign by frozen section and 1 patient diagnosed as benign had discordance with the final histopathology, which was finally diagnosed as a functional ovarian cyst (Table 1).

Of the seven borderline tumors, four patients had discordance with the final pathology. Among the four patients with discordance, two were misinterpreted as benign and later proved to be borderline ovarian tumor and two patients diagnosed as borderline tumor of ovary (BTO) by frozen were ultimately malignant on final histopathology. Among the 22 malignant patients only 2 patients
${ }^{1-4}$ Department of Surgical Oncology, Government Kilpauk Medical College, Chennai, Tamil Nadu, India

Corresponding Author: Subbiah Shanmugam, Department of Surgical Oncology, Government Kilpauk Medical College, Chennai, Tamil Nadu, India, e-mail: subbiahshanmugam67@gmail.com

How to cite this article: Shanmugam S, Govindasamy G, Susikar S, et al. Frozen Section as a Guide in Intraoperative Decision-making in the Management of Adnexal Mass Lesions. J South Asian Feder Menopause Soc 2019;7(2):66-67.

Source of support: Nil

Conflict of interest: None

had discordance. Overall, the accuracy rate of frozen was $84 \%$. The sensitivity of frozen in diagnosing benign tumors was $100 \%$, borderline was $40 \%$, and malignant tumors was $94 \%$; the specificity was $75 \%, 50 \%$, and $80 \%$; and the accuracy rate was $94 \%, 42 \%$, and $90 \%$, respectively (Table 2 ).

\section{Discussion}

Ovarian cancer accounts for $4 \%$ of cancers occurring in women. Ovarian cancer accounts for most of the gynecological cancer mortality next only to cancer cervix in India. ${ }^{1}$ The majority of women

Table 1: Comparison of frozen section with final histopathology

\begin{tabular}{lllll}
\hline S. no. & Type of tumor & Total number & Concordance & Discordance \\
\hline 1 & Benign & 17 & 16 & 1 \\
2 & Borderline & 7 & 3 & 4 \\
3 & Malignant & 22 & 20 & 2 \\
\hline
\end{tabular}

Table 2: Sensitivity, specificity, and accuracy of frozen section in various ovarian tumors

\begin{tabular}{lllll}
\hline S. no. & Type of tumor & Sensitivity (\%) & Specificity (\%) & Accuracy (\%) \\
\hline 1 & Benign & 100 & 75 & 94 \\
2 & Borderline & 40 & 50 & 42 \\
3 & Malignant & 94 & 80 & 90 \\
\hline
\end{tabular}

o The Author(s). 2019 Open Access This article is distributed under the terms of the Creative Commons Attribution 4.0 International License (https://creativecommons. org/licenses/by-nc/4.0/), which permits unrestricted use, distribution, and non-commercial reproduction in any medium, provided you give appropriate credit to the original author(s) and the source, provide a link to the Creative Commons license, and indicate if changes were made. The Creative Commons Public Domain Dedication waiver (http://creativecommons.org/publicdomain/zero/1.0/) applies to the data made available in this article, unless otherwise stated. 
with epithelial ovarian cancer have vague and nonspecific pelvic, abdominal, and menstrual symptoms. Ovarian epithelial cancers must be differentiated from benign, BTO, and functional cyst of ovaries.

Ultrasonographic signs of malignancy include an adnexal pelvic mass with areas of complexity and multiple echogenic patterns. An elevated CA-125 is not specific to ovarian cancer and is seen in diverse group of conditions. The accepted cutoff value is $35 \mathrm{U} / \mathrm{dL}$. A number of benign gynecological conditions, such as endometriosis, fibroids, infections, and pelvic inflammatory disease, may increase the CA-125 levels.

The risk of malignancy index (RMI ${ }^{2}$ incorporates the menopausal status, an ultrasonic score, and the serum CA-125 level. With an RMI score of more than 200, laparotomy and frozen section analysis should be done. For benign pathology, treatment should be individualized, limited staging for BTO, and full surgical staging for invasive ovarian cancers.

Thus, frozen section study plays an important role as a guide in intraoperative decision-making in adnexal mass lesions. But the real question is how far the frozen section can be relied upon in decision-making. The diagnostic accuracy varied from $86 \%$ to $100 \%{ }^{3}$ The overall accuracy in our setting was $84 \%$. The accuracy rates were more than $90 \%$ for benign and malignant lesions but accuracy rates drastically fell to $42 \%$ for BTO.

Also, as per other series the majority of the discordance was for mucinous and borderline tumors. ${ }^{3}$ The diagnostic accuracy of the frozen section in borderline ovarian tumors has been less well characterized with the reported rates ranging from $56 \%$ to $89 \% .{ }^{4}$ A diagnosis of BTO cannot be made prior to surgery and histopathological examination. Intraoperative decision regarding the surgical management is thus based on the frozen section. Benign tumor reported as BTO may result in unnecessary surgery and also BTO reported as malignant may result in an unnecessary staging procedure.

Over a period of time, a lot had been tried to improve the accuracy of the frozen section in BTO. A combined preoperative assessment through serum markers and ultrasonographic features may potentially reduce the risk of under diagnosis of BTOs on the frozen section while likely increasing the concomitant incidence of false-positive events. ${ }^{5}$ The common explanation for decreased accuracy given is inadequate sampling. It is also said that mucinous tumors contain benign, borderline, and malignant components in different areas in the same tumor. Adequate sampling of representative areas ${ }^{4}$ and proper communication with the pathologist may improve the results of the frozen section.

From clinical perspective, the important question is whether or not to abort the surgical procedure after a frozen diagnosis of BTO and wait for a definitive pathological diagnosis. But, it is prudent to postpone the definitive surgical management of BTO until a final histopathology is available. ${ }^{4}$

\section{Conclusion}

Frozen section is reliable in diagnosing benign and malignant ovarian tumors but not so in diagnosing borderline ovarian tumors. It is a guide to decide the extent of surgical management in benign and malignant tumors intraoperatively. The oncosurgeon has to keep in mind the possibility of invasive component when a diagnosis of borderline ovarian tumor is given by the frozen section, and it may be prudent to postpone the definitive procedure until final histopathology is available.

\section{References}

1. http://cancerindia.org.in/cp/index.php/know-about-cancer/ statistics.

2. Jacobs I, Oram D, Fairbanks J, et al. A risk of malignancy index incorporating CA-125, ultrasound and menopausal status for the accurate preoperative diagnosis of ovarian cancer. $\mathrm{Br} J$ Obstet Gynaecol 1990;97(10):922-929. DOI: 10.1111/j.1471-0528.1990. tb02448.x.

3. Da Silva RDP, Souto LRM, de Macedo Matsushita G, et al. Diagnostic accuracy of frozen section tests for surgical diseases. Rev Col Bras Cir 2011;38(3):149-154.

4. Tempfer CB, Polterauer S, Bentz E-K, et al. Accuracy of intraoperative frozen section analysis in borderline tumors of the ovary: a retrospective analysis of 96 cases and review of the literature. Gynecol Oncol 2007;107(2):248-252. DOI: 10.1016/j.ygyno.2007.06.008.

5. Gizzo S, Berretta R, Di Gangi S, et al. Borderline ovarian tumors and diagnostic dilemma of Intraoperative diagnosis: could preoperative $\mathrm{He}_{4}$ assay and ROMA score assessment increase the frozen section accuracy? A multicenter case-control study. Biomed Res Int 2014 803598. DOI: 10.1155/2014/803598. 